\title{
DESENVOLVIMENTO DE MÉTODO SIMPLES PARA A DETERMINAÇÃO DE RESÍDUOS DE DIURON POR CROMATOGRAFIA A LÍQUIDO EM AMOSTRAS DE LARANJA
}

\author{
OZELITO POSSIDÔNIO DE AMARANTE JUNIOR* \\ NATILENE MESQUITA BRITO** \\ MARIA LÚCIA RIBEIRO***
}

\begin{abstract}
Este artigo apresenta método simples para a extração e determinação de diuron em amostras de laranja (compreendendo casca e polpa). O método consistiu na extração do pesticida com acetona em agitador mecânico. O extrato foi filtrado em Celite® e particionado com n-hexano. Evaporou-se a fase hexânica sob $\mathrm{N}_{2}$, sendo o resíduo solubilizado em metanol. A análise foi realizada por Cromatografia a Líquido de Alta Eficiência (CLAE) com detecção por Ultra-violeta (UV) em $230 \mathrm{~nm}$. Obtiveram-se recuperações médias entre 109 e $110 \%$ para amostras fortificadas nos níveis 0,1 e $0,5 \mathrm{mg} \mathrm{Kg}^{-1}$. Os Limites de Quantificação (LQ) e de Detecção (LD) foram $0,1 \mathrm{mg} \mathrm{Kg}^{-1}$ e $0,004 \mathrm{mg} \mathrm{Kg}^{-1}$, respectivamente. As amostras analisadas não apresentaram resíduos de diuron nas condições experimentais estudadas.
\end{abstract}

* Doutor em Ciências da Engenharia Ambiental, Professor do Departamento Acadêmico de Química, Centro Federal de Educação Tecnológica do Maranhão (CEFET-MA) São Luís-MA (e-mail: ozelito@cefetma.br).

* Doutora em Química Analítica, Professora do Departamento Acadêmico de Química, CEFET-MA (email: natilene@cefet-ma.br).

** Doutor em Engenharia Agronômica, Pesquisador Embrapa Meio Ambiente, Jaguariúna, SP.

*** Doutora em Química Orgânica, Professora do Departamento de Química Orgânica, Instituto de Química, Universidade Estadual Paulista (UNESP), Araraquara - SP. 


\section{INTRODUÇÃO}

Os herbicidas, utilizados pela Inglaterra na Guerra das Malvinas, foram extensamente empregados na Guerra do Vietnã pela força aérea norte-americana (AMARANTE JÚNIOR et al., 2003 e 2002). Desde então, esses compostos vêm sendo aplicados na substituição da capina mecânica, observandose elevado crescimento na produção e comercialização de diferentes princípios ativos para essa finalidade.

O uso de herbicidas leva à exposição da população que habita próximo aos locais em que são produzidos ou aplicados, bem como daqueles que consomem os produtos das lavouras tratadas com tais compostos (POZZEBON et al., 2003). O diuron [3-(3,4-diclorofenil)-1,1-dimetiluréia], s-triazina, destaca-se entre os herbicidas por ser amplamente aplicado no controle de ervas em campos de cultivo de citrus (BARREDA JÚNIOR et al., 1998). Suas propriedades físicas e químicas incluem solubilidade em água de $42 \mathrm{mg} / \mathrm{L}$ em 25ํㅡ, não-ionizável, não-volátil (SOUZA, BOEIRA e GOMES, 2000) e tempo de meia-vida no solo ( $\mathrm{DT}_{50}$ ) de $328+212$ dias (RAO e DAVIDSON, 1982). A fórmula estrutural desse composto é apresentada na Figura 1. Grandes áreas de cultivo de laranja são encontradas na região de Araraquara (SP), o que torna relevante a investigação da presença de resíduos de diuron em frutos comercializados nessa cidade.

\section{FIGURA 1 - FÓRMULA ESTRUTURAL DO DIURON}

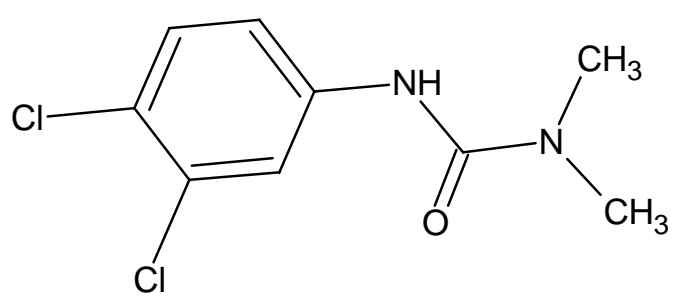

Dentre os diversos métodos encontrados na literatura para a determinação de diuron pode-se citar a análise direta em solução aquosa por Cromatografia a Líquido de Alta Eficiência (CLAE), usando coluna C18, eluição com metanol/água (63:37, v/v) e detecção em 251 nm (SOUZA, BOEIRA e GOMES, 2000). RUBERU, DRAPER e PEREIRA (2000) empregaram sistema tampão de fosfato dibásico de potássio com acetonitrila na proporção $60: 40(\mathrm{v} / \mathrm{v})$ como fase móvel e fluxo de 0,4 $\mathrm{mL} / \mathrm{min}$. Também utilizaram fase móvel composta pelo mesmo tampão e metanol na proporção 50:50 (v/v), com fluxo de $0,8 \mathrm{~mL} / \mathrm{min}$. Eluição com sistema gradiente de acetonitrila/água foi citada para a determinação multirresidual de feniluréias com detecção em $245 \mathrm{~nm}$ (CURINI et al., 2000). Alguns estudos empregaram a detecção por espectrometria de massas (VAN DER HEEFT et al., 2000; PEÑA, MAHEDERO e BAUTISTA-SÁNCHEZ, 2003), sendo citada a detecção por fluorescência induzida (PEÑA, MAHEDERO e BAUTISTA-SÁNCHEZ, 2002). MORAES et al. (2002) determinaram várias feniluréias, incluindo o diuron, por cromatografia em camada delgada (CCD) e detecção por inibição da fotossíntese (reação de Hill).

Apesar da ampla utilização de diuron em campos de cultivos de frutas cítricas, especialmente nas plantações de laranjas, não se encontrou na literatura pesquisada método específico para amostras de laranja.

Este trabalho teve por objetivo desenvolver método cromatográfico para a determinação de resíduos de diuron em frutos inteiros (casca e polpa) de laranjas, obtidas no comércio de Araraquara durante 0 ano de 2002. 


\section{MATERIAL E MÉTODOS}

\subsection{SISTEMA DE CROMATOGRAFIA A LÍQUIDO (CL)}

O sistema CL (Waters, Milford, MA, USA) consistiu de dispositivo de controle de solventes (Modelo 501), com detector de Ultra-violeta (UV) e comprimento de onda variável (Modelo 486), injetor Rheodyne ${ }^{\circledR}$ e integrador (Modelo 746). Empregou-se coluna LiChrospher 100 RP-18 (125 x 4 mm, $5 \mu \mathrm{m}$ ), acoplada em pré-coluna do mesmo material (ambas Merck). As análises foram realizadas em $230 \mathrm{~nm}$, usando-se fase móvel no regime isocrático com metanol/água (60:40) e fluxo de 0,8 mL/min. Todos os solventes empregados na fase móvel foram previamente filtrados e desgaseificados por aplicação de ultra-som.

\subsection{REAGENTES E SOLUÇÕES PADRÃO}

Foram utilizados metanol, acetona e n-hexano (Mallinckrodt) de grau cromatográfico e padrão de referência de diuron obtido do Dr. Ehrenstorfer (Alemanha). Soluções estoque foram preparadas pela dissolução desse composto em metanol, obtendo-se soluções-padrão pela diluição das soluções estoque em metanol, as quais foram estocadas a $-18^{\circ} \mathrm{C}$ até seu uso.

\subsection{PREPARAÇÃO DAS AMOSTRAS, FORTIFICAÇÃO E EXTRAÇÃO}

Amostras de laranja, adquiridas no comércio de Araraquara, foram cortadas com faca de aço inoxidável e processadas em blender para obtenção de pasta homogênea. Efetuou-se a fortificação das amostras pela adição de volume adequado de solução estoque em 10,0 g da pasta homogênea. As amostras fortificadas e não-fortificadas foram tratadas com $10 \mathrm{~mL}$ de acetona. Extraiu-se o pesticida por 30 minutos em mesa agitadora. Filtrou-se o extrato em $1 \mathrm{~g}$ de Celite $\AA$, usando funil de vidro com ponta de fibra de vidro silanizada. Realizou-se partição com n-hexano em duas etapas para a purificação do extrato, descartando-se a fase hexânica. O extrato em acetona foi evaporando até $1 \mathrm{~mL}$ em evaporador rotatório e seco sob suave corrente de $\mathrm{N}_{2}$, retomando-se o resíduo com metanol ( $1 \mathrm{~mL}$ ).

\subsection{ANÁLISE POR CROMATOGRAFIA A LÍQUIDO}

Os extratos e soluções-padrão $(20 \mu \mathrm{L})$ foram injetados no sistema cromatográfico, escolhendose o intervalo de trabalho de 0,5 a $10,0 \mathrm{mg} \mathrm{L}^{-1}$. As recuperações foram calculadas para amostras de laranja fortificadas com 0,1 e $0,5 \mathrm{mg} \mathrm{Kg}^{-1}$ de diuron, com cinco replicatas para cada nível. Determinouse o limite de detecção (LD) mediante o cálculo estatístico proposto por Thier e Zeumer (BRITO et al., 2001; THIER e ZEUMER, 1987). Considerou-se o menor nível de fortificação como limite de quantificação (LQ), uma vez que essa é a menor concentração em que são conhecidas a exatidão e a precisão do método (AMARANTE JÚNIOR et al., 2001; THIER e ZEUMER, 1987).

\section{RESULTADOS E DISCUSSÃO}

Buscando otimizar método para a determinação de diuron em amostra de laranjas que pudesse ser empregado com diferentes propósitos (determinação do pesticida em frutos tipo exportação e estudos de campo, entre outros) foi necessário estudar o fruto inteiro (polpa e casca). Entretanto, os extratos em acetona apresentaram grande quantidade de co-extrativos não permitindo a separação cromatográfica do analito investigado.

Com o intuito de eliminar os co-extrativos foram realizados os seguintes testes: i) filtração do extrato em Celite $\AA$; ii) filtração do extrato em Celite $\AA$ e purificação por extração em fase sólida (SPE) 
com $\mathrm{C}_{18}$; iii) filtração do extrato em Celite $\AA$ e partição em duas etapas com n-hexano. De todas essas tentativas, a última foi a única que permitiu a análise cromatográfica do princípio ativo. No entanto, o cromatograma ainda apresentou diversos componentes da amostra.

Os cromatogramas obtidos para a amostra testemunha (sem o pesticida) e as amostras fortificadas são apresentados na Figura 2. Verificou-se a complexidade da matriz pelos vários picos apresentados, mesmo após filtração com Celite $\AA$. A análise tornou-se possível pela inexistência de picos de co-extrativos no mesmo tempo de retenção do diuron, como observado no cromatograma da amostra testemunha.

No gráfico analítico, apresentado na Figura 3, pode-se observar pequena dispersão dos sinais em torno da reta obtida pela regressão, bem como excelente relação linear evidenciada pelo coeficiente de correlação $\left(R^{2}=0,9994\right)$. O método apresentou-se ainda bastante sensível. A sensibilidade, definida como o aumento no sinal causado por pequeno incremento no valor da concentração (AMARANTE JÚNIOR et al., 2001; THIER e ZEUMER, 1987), é obtida pelo coeficiente angular da reta (BRITO et al., 2002).

\section{FIGURA 2 - CROMATOGRAMAS OBTIDOS PARA AS AMOSTRAS TESTEMUNHA (A), FORTIFICADA COM 0,1 (B) E $0,5 \mathrm{mg} \mathrm{kg}^{-1}$ (C) \\ B}

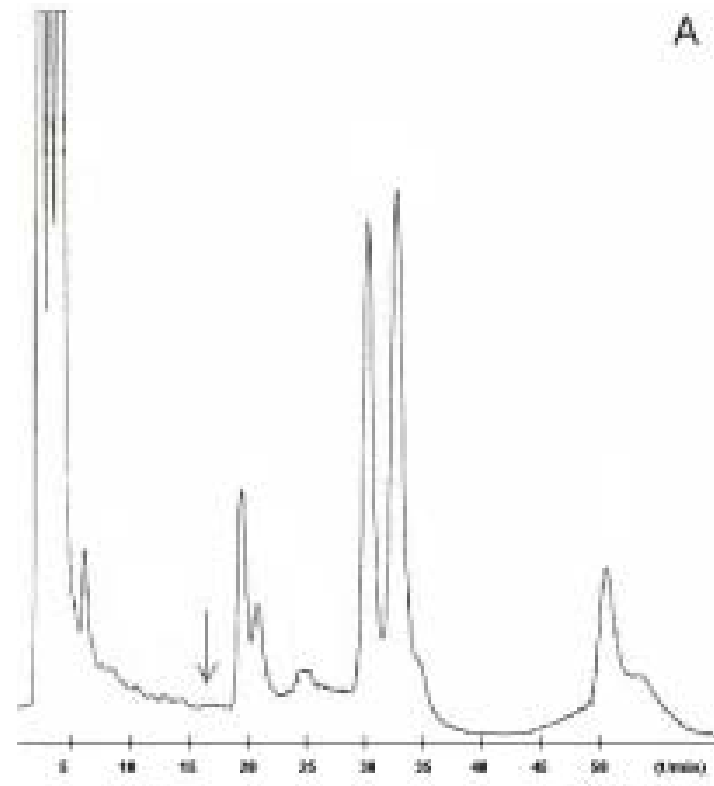

A
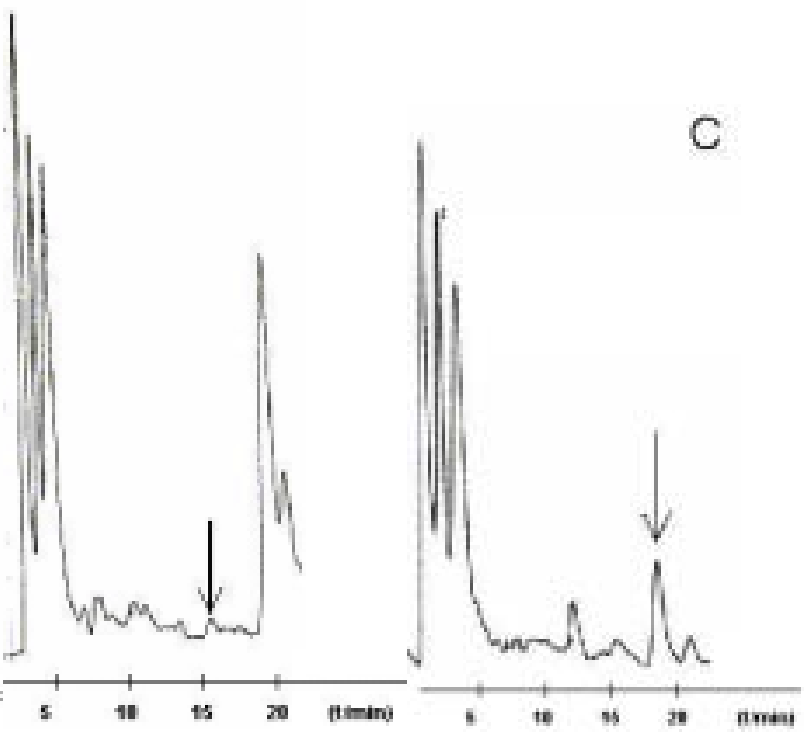

FIGURA 3 - GRÁFICO ANALÍTICO DO PESTICIDA DIURON OBTIDO PELA INJEÇÃO DE $20 \mu \mathrm{L}$ DAS SOLUÇÕES DE TRABALHO EM SISTEMA CROMATOGRÁFICO COM COLUNA C18, ELUIÇÃO COM METANOL/ÁGUA (60:40), 0,8 mL/min E DETECÇÃO EM $230 \mathrm{~nm}$

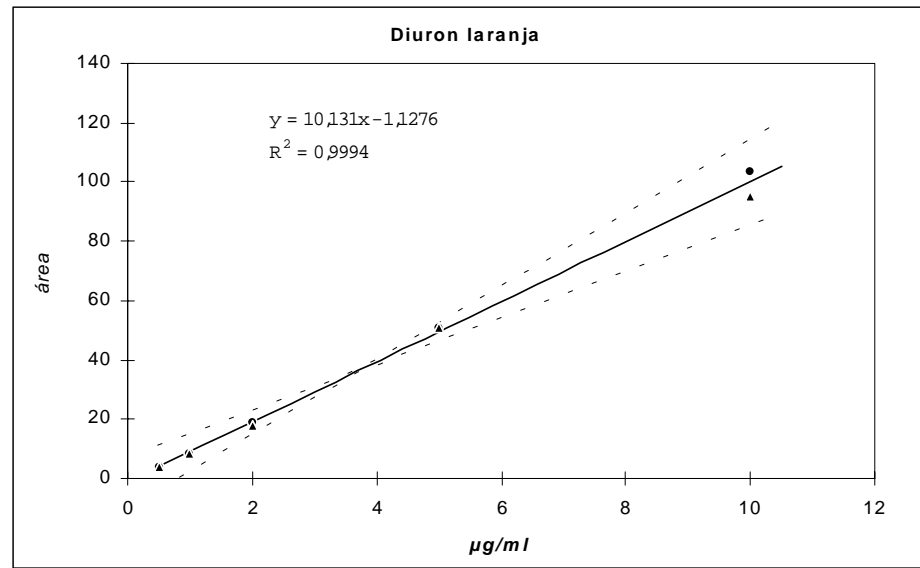


Os resultados de recuperação (Tabela 1) expressam a exatidão do método. Os valores foram considerados adequados, visto que permaneceram entre o intervalo recomendado (70 e 120\%) para a análise de resíduos de pesticidas (SOUZA, BOEIRA e GOMES, 2000; RAO e DAVIDSON, 1982). Avaliou-se a precisão pelo coeficiente de variação $(\mathrm{CV})$ ou desvio-padrão relativo, sendo considerado preciso o método que obtém CV inferior ou igual a 20\% (SOUZA; BOEIRA e GOMES, 2000; RAO e DAVIDSON, 1982).

TABELA 1 - RECUPERAÇÕES DE DIURON EM AMOSTRAS DE LARANJA FORTIFICADAS

\begin{tabular}{l|c|c|c}
\hline & $\begin{array}{c}\text { Nível de fortificação } \\
(\mu \mathrm{g} / \mathrm{g})\end{array}$ & $\begin{array}{c}\text { Recuperação } \\
(\%)\end{array}$ & $\begin{array}{c}\text { C V } \\
(\%)\end{array}$ \\
\hline DIURON & 0,1 & 109 & 7 \\
& 0,5 & 110 & 15 \\
\hline
\end{tabular}

$\mathrm{n}=$ cinco determinações.

$\mathrm{CV}=$ coeficiente de variação.

O Limite de Detecção (LD), calculado de acordo com THIER e ZEUMER (1987) foi de 0,04 $\mu \mathrm{g} /$ g e o Limite de Quantificação (LQ) de 0,1 $\mu \mathrm{g} / \mathrm{g}$ (menor nível de fortificação). Esses valores apresentaramse bastante satisfatórios para análises por Cromatografia a Líquido, principalmente pela complexidade da amostra. Esse método, considerado validado, foi empregado para a análise de 20 amostras de laranjas coletadas no comércio de Araraquara durante o ano de 2002. Nenhuma das amostras investigadas apresentou resíduos de diuron sob as condições analíticas empregadas.

\title{
4 CONCLUSÃO
}

O método de determinação de diuron em amostras de laranja mostrou-se bastante simples e eficiente, consistindo de extração em duas etapas, seguida de filtração, partição líquido-líquido, evaporação e análise por Cromatografia a Líquido. O cromatograma apresentou perfil bastante complexo, indicando extrato com diversos compostos, o que não inviabilizou a análise pela ausência de coeluição no tempo de retenção da molécula de interesse. Os resultados obtidos no estudo de recuperação demonstraram que o método é exato e preciso, podendo ser empregado na análise de laranja para a determinação de resíduos desse pesticida. Os Limites de Detecção e Quantificação foram suficientemente baixos, garantindo a confiabilidade dos resultados. Todas as amostras obtidas no comércio da cidade de Araraquara, durante o ano de 2002, submetidas ao referido método não apresentaram concentrações superiores ao LD do método.

\begin{abstract}
DEVELOPMENT OF SIMPLE METHOD FOR DETERMINATION OF DIURON RESIDUES BY LIQUID CHROMATOGRAFY IN ORANGE SAMPLES

This paper presents a simple method for extraction and determination of diuron in orange samples (enclosed pulp and peel). The method consisted of the pesticide extraction with acetone on mechanical agitator system. The extract was filtrated in Celite ${ }^{\circledR}$ and partitioned with $\mathrm{n}$-hexane. The hexane phase was evaporated and the residue dissolved in methanol. High Performance Liquid Chromatography (HPLC) with UV detection at $230 \mathrm{~nm}$ was used for analysis. Average recoveries were obtained between 109 and $110 \%$ for samples fortified at 0.1 and $0.5 \mathrm{mg} \mathrm{Kg}^{-1}$ levels. The Limits of Quantification (LOQ) and Limit of Detection (LOD) were $0.1 \mathrm{mg} \mathrm{Kg}^{-1}$ and $0.004 \mathrm{mg} \mathrm{Kg}^{-1}$, respectively. The samples analyzed have not shown Diuron residues in the studied experimental conditions.
\end{abstract}

KEY-WORDS: PESTICIDE; CITRIC FRUITS; HPLC; DIURON. 


\section{REFERÊNCIAS}

1 AMARANTE JÚNIOR, O. P. de; SANTOS, T. C. R. dos; NUNES, G. S.; RIBEIRO, M. L. Breve revisão de métodos de análise do herbicida ácido 2,4-diclorofenoxiacético (2,4-D). Quím. Nova, v.26, n.2, p. 223-229, 2003.

2 AMARANTE JÚNIOR, O. P. de; SANTOS, T. C. R. dos; BRITO, N. M.; RIBEIRO, M. L. Revisão das propriedades, usos e legislação do ácido 2,4-diclorofenoxiacético (2,4-D). Cad. Pesq., v. 13, n.1, p. 35-42, 2002.

3 POZZEBON, J. M.; QUEIROZ, S. C. N.; MELO, L. F. C.; KAPOR, M. A.; JARDIM, I. C. S. F. Application of new highperformance liquid chromatography and solid-phase extraction materials to the analysis of pesticides in human urine. J. Chromatogr. A, v. 987, p. 381-387, 2003.

4 BARREDA JÚNIOR, D. G. de; VILA, M. G.; RUEDA, E. L.; OLMO, A. S.; BARREDA, D. G. de; CUADRA, J. G. de la; TEN, A.; PERIS, C. Dissipation of some citrus selective residual herbicides in an irrigation well. J. Chromatogr. A, v. 795, p. 125-131, 1998.

5 SOUZA, M. D.; BOEIRA, R. C.; GOMES, M. A. F. Adsorção e dessorção de diuron em solos tropicais. Pesticidas: r. ecotoxicol. e meio ambiente, v. 10, p. 113-124, 2000.

6 RAO, P. S. C.; DAVIDSON, J. M. Retention and transformation of selected pesticides and phosphorus in soil-water systems: a critical review. Washington: US-EPA, 1982.

7 RUBERU, S. R.; DRAPER, W. M.; PERERA, S. K. Multiresidue HPLC methods for phenyl urea herbicides in water. J. Agric. Food Chem., v. 48, n. 9, p. 4109-4115, 2000.

8 CURINI, R.; GENTILI, A.; MARCHESE, S.; MARINO, A.; PERRET, D. Solid phase extraction followed by high-performance liquid chromatography-ionspray interface-mass spectrometry for monitoring of herbicides in environmental water. J. Chromatogr. A., v. 874, n. 2, p. 187-198, 2000.

9 VAN DER HEEFT, E.; DIJKMAN, E.; BAUMANN, R. A.; HOGENDOORN, E. A. Comparison of various liquid chromatography methods involving UV and atmospheric pressure chemical ionization mass spectrometric detection for the efficient trace analysis of phenylurea herbicides in various types of water samples. J. Chromatogr. A., v. 879, n. 1, p. 3950, 2000.

10 PEÑA, A. M. de la; MAHEDERO, M. C.; BAUTISTA-SÁNCHEZ, A. Monitoring of phenylurea and propanil herbicides in river water by solid-phase-extraction high performance liquid chromatography with photoinduced-fluorimetric detection. Talanta, v. 60, n. 2-3, p. 279-285, 2003.

11 PEÑA, A. M. de la; MAHEDERO, M. C.; BAUTISTA-SÁNCHEZ, A. High performance liquid chromatography determination of phenylureas by photoinduced-fluorimetric detection. J. Chromatogr. A., v. 950, p. 287-291, 2002.

12 MORAES, S. L.; REZENDE, M. O. O.; NAKAGAWA, L. E.; LUCHINI, L. C. Análise de resíduos de pesticidas em tomates por cromatografia em camada delgada. Quím. Nova, v. 25, n.2, p. 196-202, 2002.

13 BRITO, N. M.; AMARANTE JUNIOR, O. P. de; POLESE, L.; SANTOS, T. C. R. dos; RIBEIRO, M. L. Avaliação da exatidão e da precisão de métodos de análise de resíduos de pesticidas mediante ensaios de recuperação. Pesticidas: $r$. ecotoxicol. e meio ambiente, v. 12, p. 155-168, 2002.

14 AMARANTE JUNIOR, O. P. de; CALDAS, E. P. A.; BRITO, N. M.; SANTOS, T. C. R. dos; VALE, M. L. B. F. Validação de métodos analíticos: uma breve revisão. Cad. Pesq., v. 12, n.1/2, p. 116-131, 2001. 